\title{
SOBRE LAS CAPITULACIONES MATRIMONIALES DE FRANCISCO DE GOYA Y LA PRISA DEL ARAGONÉS EN ABANDONAR ROMA
}

\author{
RaQuel Gallego \\ Doctora en Historia del Arte, ACAF/ART, Universitat de Barcelona
}

\begin{abstract}
El hallazgo de las capitulaciones matrimoniales de Francisco de Goya ha servido, entre otras cosas, para despejar algunas de las dudas existentes en torno a la permanencia del pintor aragonés en Italia. El estudio pormenorizado de uno de los documentos que las integran, que no ha sido transcrito hasta el momento, podría arrojar interesantes datos acerca de la premura con que debió tener lugar el regreso del pintor aragonés a España y de la manera en que Juan Adán y Manuel Eraso dieron su testimonio para que se pudiese celebrar el enlace entre Goya y Josefa Bayeu. Este artículo se enmarca en una de las líneas de investigación del Proyecto ACAF/ART (HAR2009-07053) y se ha llevado a cabo bajo su patrocinio.
\end{abstract}

Palabras clave: Goya; Italia; Adán; Eraso; Capitulaciones matrimoniales.

\section{ON FRANCISCO DE GOYA'S MARRIAGE CONTRACT AND HIS RUSH TO LEAVE ROME}

The discovery of the marriage contract of Francisco de Goya has helped to clarify, among other things, certain aspects of the presence of the Aragonese painter in Italy. A detailed analysis of one of the documents in the dossier, not transcribed until now, throws new light on the reasons for Goya's haste to return to Spain, as well as on the role played by Juan Adán and Manuel Eraso in facilitating the wedding of the artist with Josefa Bayeu. This article is part of one of the lines of research for the project ACAF/ART (HAR200907053), and has been developed under its sponsorship.

Key words: Goya; Italy; Adán; Eraso; Marriage contract.

En el número 44 del Boletín del Museo del Prado, Jesús López Ortega publicó la transcripción de la mayor parte de la documentación que integra el expediente de las capitulaciones matrimoniales de Francisco de Goya (Fuendetodos, Zaragoza, 1746-Burdeos, 1828), acompañada de un breve comentario crítico ${ }^{1}$. Esta documentación recoge que, en el año 1773, el joven pintor

\footnotetext{
${ }^{1}$ López OrTega, 2008:62-68. El documento que se conserva en el Archivo Histórico Diocesano de Madrid es el "Expediente matrimonial de Francisco de Goya y Josefa Bayeu", Sección Protocolos, Notario Nicolás de la Fuente, 15-7-1773/19-7/1773, sign. 4553/7.
} 
aragonés contrajo matrimonio, en la parroquia de San Martín de Madrid, con María Josefa Bayeu y Subías, hermana de los también pintores Francisco (Zaragoza, 1734-Madrid, 1795) y Ramón (Zaragoza, 1746-Aranjuez, 1793) e hija de Ramón y María. Para la celebración del enlace matrimonial, el notario Nicolás de la Fuente abrió un expediente el 15 de julio de 1773 que él

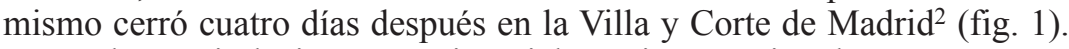

En las capitulaciones matrimoniales existen varios documentos que contienen datos fundamentales para conocer y acotar la estancia en Italia del pintor aragonés ${ }^{3}$, de la que hemos comenzado a vislumbrar algunas de sus características durante los últimos años ${ }^{4}$. Gracias a tales documentos, se puede dar por zanjada la antigua polémica relativa a la duración del soggiorno italiano de Goya; en estos momentos, quedan pocas dudas de que el viaje a Italia tuvo lugar en algún momento del año 1769 , probablemente durante la primavera, mientras que el regreso a España se habría podido producir en la primavera de $1771^{5}$.

Probablemente, el más relevante de los documentos de las capitulaciones matrimoniales que se refieren a la estancia de Goya en Italia, es el que tiene el número 6 en el apéndice documental del artículo de Jesús López ${ }^{6}$, la declaración realizada por Juan Adán (Tarazona, 1741-Madrid, 1816) ${ }^{7}$ y Manuel Eraso ${ }^{8}$ (Zaragoza, 1742-Burgos, 1813). Está fechado el 27 de abril de 1771 en

2 LÓPEZ OrTEGA, 2008: 66. María Josefa Bayeu, de veinticinco años de edad en el momento del matrimonio, era natural de Zaragoza, aunque en 1773 residía en la casa de su padre en Madrid desde hacía once años. Ésta se había prometido con Goya un año antes y el pintor, para poder contraer matrimonio, aportó, según era costumbre, la documentación que demostraba que reunía las condiciones requeridas. Teresa Ens, mujer del dorador José Escorción, prestó declaración en favor de Josefa Bayeu ante el notario José Antonio Jiménez. Ésta residía en Madrid y conocía a la hija de Bayeu desde que la futura esposa llegó a la capital. También declaró en su favor Bernardina de Villanueva, mujer de Francisco Berrueta, domiciliada en Madrid, que mantenía una relación con la futura esposa desde hacía diez años.

${ }^{3}$ López Ortega, 2008: 65, 67. En el documento número 1 se dice que Goya estuvo en Roma cuatro años antes de casarse, en donde realizó lo que se denomina "sus diligencias de libertad", mediante las que acredita no haber contraído matrimonio durante su permanencia en la ciudad eterna. En el documento número 7 se apunta que el pintor aragonés residió en Zaragoza hasta 1769, año en que se fue a Roma en donde estudió en la Academia del Disegno y en donde permaneció durante dos años.

${ }^{4}$ Gallego, 2011: 45-59. Mena, 1994. Mena, 2010: 353-366. Navarrete, 2002: 293-296. Reuter, 2003: $37-48$. Sureda, 2008. Tosini Pizzetti, 2006. Urrea, 1993: 59-66.

5 Ona GonzÁlez, 1997: 75-86. Gracias a los estudios de José Luis Ona a partir de las matrículas pascuales, sabemos que Goya se localiza en Zaragoza, al menos hasta la Pascua de 1769. En los años 1767, 1768 y 1769, el pintor aragonés está establecido en esta ciudad; durante los dos primeros años en la parroquia de la Magdalena, viviendo en Puerta Quemada, y en 1769 se trasladó a la parroquia de San Miguel de los Navarros, puesto que el pintor se alojaba con su familia en la Baxada del Gastón. Sin embargo, en 1770 la familia de Goya sigue viviendo en la Baxada del Gastón, en la parroquia de San Miguel de los Navarros, en donde no se constata ya la presencia del joven artista. De la misma forma, el pintor no es registrado en el año 1771 en la demora familiar que, en ese año, se encuentra en el número 70 de Puerta Quemada.

${ }^{6}$ LÓPEZ OrTEGA, 2008: p. 67.

7 Azcue Brea, 1994: 222-246. Calvo Carretero, 2013. Pardo Canalis, 1951: 172. Pardo Canalis, 1965/66: 197. Pardo Canalis, 1971:435-436. Sureda, 2008: I 249. Urrea, 2006: 264-266. Vaquero PelÁez, 1992: 547-562. En 1755, Juan Adán abandonó su Tarazona natal para dirigirse a Zaragoza, en donde comenzó un periodo formativo en el taller de José Ramírez de Arellano (Bells, Huesca, 1705-Zaragoza, 1770). En 1765, estaba en Italia, si bien no debiéramos excluir la posibilidad de que llegase allí algunos años antes, quizá en compañía de su amigo y colega Manuel Eraso. En 1767 consiguió una ayuda extraordinaria de la Real Academia de Bellas Artes de San Fernando para continuar su formación en la ciudad eterna que debería habérsele retirado en 1775 aunque, gracias a la intercesión de otro aragonés, José Nicolás de Azara (Barbuñales, Huesca, 1730-París, 1804), se le prorrogó durante otros dos años mas. En realidad, la disfrutó hasta 1776 porque precisamente en aquel año, fue reclamado por el obispo de Lérida para trabajar en la catedral.

8 IBÁÑEZ PÉREZ, 1982: 173-178. SuREDA, 2008: I 258-259. UrREA, 2006: 197-198. Manuel Eraso, en un primer momento estudiante de arquitectura, se formó en Zaragoza probablemente bajo las enseñanzas de José Luzán (Zaragoza, 1710-Zaragoza, 1785) o de Juan Andrés Merklein (ANHoLt, Flandes?-Zaragoza, 1797) desde donde, en el año 1761,

Arch. esp. arte, LXXXVII, 346, ABRIL-JUNIO 2014, 109-118 ISSN: 0004-0428, eISSN: 1988-8511, doi: 10.3989/aearte.2014.08 


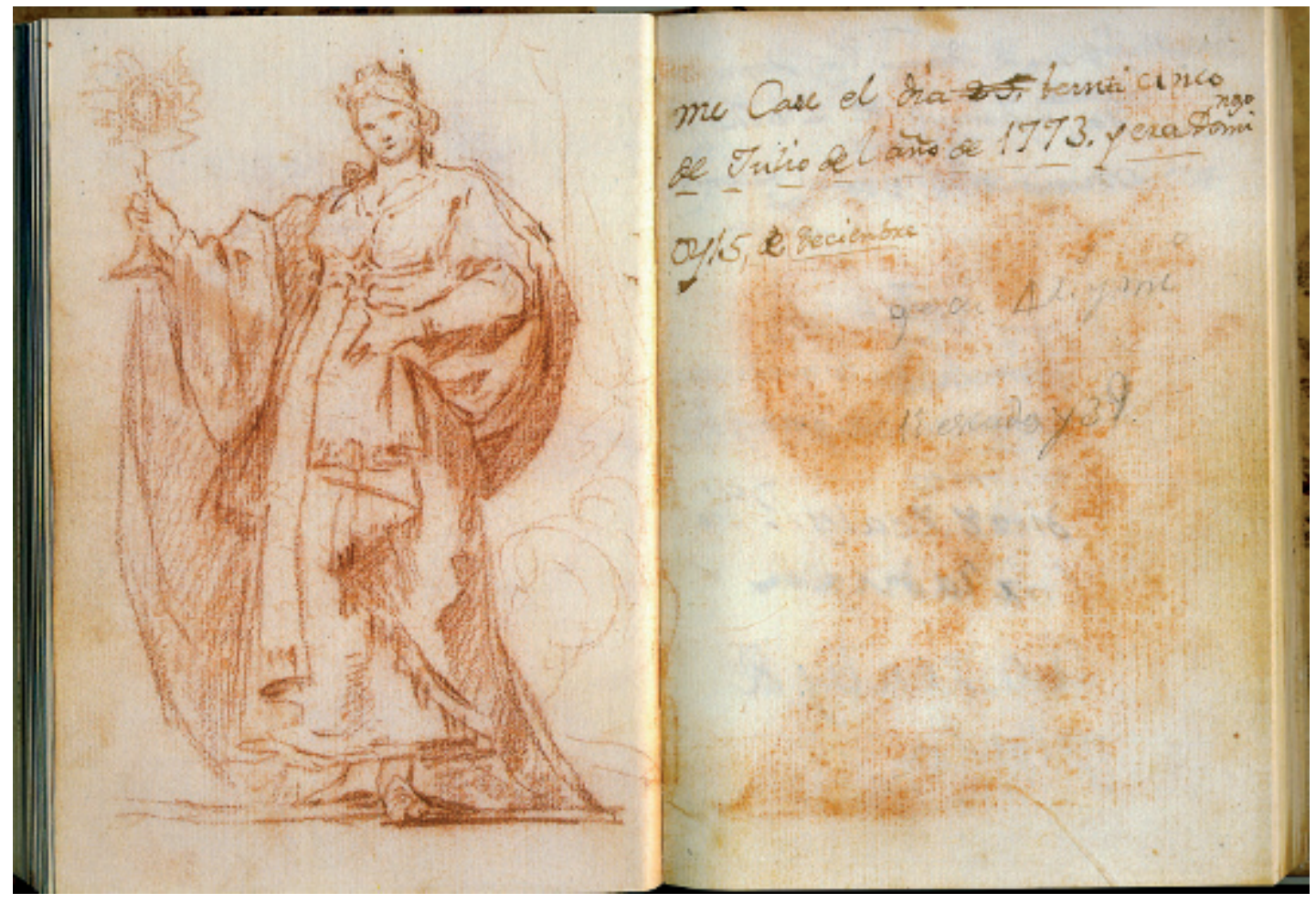

Fig. 1. Francisco de Goya, Cuaderno italiano, pp. 88r-89a, 1771-1788, Museo Nacional del Prado, Madrid.

la ciudad eterna y en él ambos afirman conocer sobradamente a Francisco de Goya, con el que compartieron la estancia en Roma, en donde el joven pintor se ejercitó en la pintura y en donde no había contraído matrimonio. Este documento concede total validez a la hipótesis de Arturo Ansón Navarro ${ }^{9}$, sobre la que se reflexionó en el catálogo de la exposición Goya e Italia ${ }^{10}$, acerca de la relación que Goya podría haber entablado en Zaragoza con sus dos paisanos ${ }^{11}$ y que habría mantenido durante el tiempo que permaneció en Italia.

se fue a Italia. En 1763, llegaron a Madrid procedentes de Roma algunas obras realizadas por Manuel Eraso junto a las que enviaban los pensionados de la Real Academia de Bellas Artes de San Fernando, necesarias para la adecuada evaluación del desarrollo de su formación en la ciudad eterna. Gracias a la intercesión de Azpuru consiguió, en 1766, una ayuda extraordinaria de la institución académica madrileña que le permitió permanecer en Italia hasta 1773, posiblemente la fecha en que se produjo su regreso a España.

9 ANSÓN NAVARro, 1995: 82.

10 Gallego, 2008: 48-51.

11 Ansón NAVARro, 1996: 57-58, 60. Cuando contaba con 13 años de edad, es decir, en 1759, Francisco de Goya, según señala su hijo Javier en una breve biografía que envió a Martín Fernández Navarrete, comenzó a estudiar en la academia de José Luzán, formado en el mundo pictórico napolitano. Probablemente, el de Fuendetodos tuvo un acceso privilegiado al estudio de Luzán ya que su padre, José, era amigo de éste. Los hermanos doradores de José Luzán, Juan y Pedro, habían trabajado con el padre de Goya en algunos encargos. Asimismo, tal y como apunta Arturo Ansón, el joven pintor podría haber asistido a las clases nocturnas que Luzán impartía en la Academia de Dibujo de Zaragoza, creada por la Primera Junta Preparatoria en 1754 gracias al impulso de algunos de los más relevantes nobles de la

Arch. esp. arte, LXXXVII, 346, ABRIL-JUNIO 2014, 109-118

ISSN: 0004-0428, eISSN: 1988-8511, doi: 10.3989/aearte.2014.08 
De la totalidad del expediente matrimonial de Goya, existe una parte que no ha sido transcrita ni estudiada: la que se enlaza con el documento número 6 del artículo de Jesús López ${ }^{12}$ mediante la última frase del recto del folio que continua en el verso del $\mathrm{mismo}^{13}$, mientras que, en otro folio en el que únicamente se ha escrito en el recto, se concluye el documento ${ }^{14}$. Todo ello se llevó a cabo ante un notario llamado Piacentino Monti ${ }^{15}$ de la Curia Cameral ${ }^{16}$ o Auditor Camerae, sita en el palacio de Montecitorio, considerado el primer tribunal del Papa ${ }^{17}$. Monti residía en la parroquia de Sant'Andrea delle Fratte, era oriundo de Sonnino, una localidad que actualmente se encuentra en la provincia de Latina, en el territorio del Lacio ${ }^{18}$, y prestó sus servicios de forma habitual en el oficio IV del mencionado Auditor Camerae entre los años 1754 y 1773. El Auditor Camerae contaba con su propio grupo de notarios ${ }^{19}$ quienes, paralelamente, podían

ciudad aragonesa. Debió ser en este ambiente donde Goya entabló la relación con Manuel Eraso y con Juan Adán y quizá, también, con José Beratón (Zaragoza, 1746-Madrid, 1796) y Joaquín Arali (Zaragoza, 1737-Madrid, 1811).

12 La transcripción completa del recto del primer folio es la siguiente: "Nosotros los abaxo firmados Certificamos y/ hacemos feé con nuestro Juramento aquien/ pertenciese como conozemos muy bien el S.or/ D.n Fran.co Goya, natural dela ciudad de Za-/ ragoza Yjo de Joseph Goya y en todo el tiem-/ po que dho D.n Fran.co à echo demora en esta/ Ciudad de Roma donde se ha exercitado en haprender el Arte dela Pintura hasta todo/ el presente dia no ha contraido Matrimonio/ ninguno ni se ha obligado tanpoco contraerl/ lo y esto lo savemos por haver tenido conti/ nua practica y hamistad con el sobre dho/ D.n Fran.co Goya. Y por ser tambien notorio/ à muchos en causa de Ziencia \&. En/ Roma yo 27 y Abril delaño 1771./Manuel Eraso Pintor pensionado para/ la Pintura por S.M.C./ Juan Adan Pe.do para la escultura por su/ M.C./ In Nomine Domini Amen/ Fidem facio per presentes Causarum Curia Camerae".

${ }^{13}$ Quisiera agradecer a Jorge Tomás García su colaboración en la transcripción del documento, así como sus sugerencias por lo que respecta a la interpretación del texto. El contenido del verso del primer folio es el siguiente: "Aplicus Notarius publicus Infrascriptus qua/ subscripta die antedicti D. Emanuel Eraso/ filius D. Iosephi de Zaragoza in Hispanis/ et D. Ioannes Adam filius alterius Ioannis/ Adam de Tarazona pariter in Hispaniis mihi/ notario optime cogniti spontes ac alia ora / medio iuramento factis recognoverunt et/ recognoscunt eorum subscriptionem per ipsorum/ in calce retroscripte fidei et attestationis/ apposit. Similique Iuramento omnia et/ singula in eodem fide et attestatione/ contenta et expressa vera fuisse et esse affi/ rmarunt et tamquam talia comprobarunt/ non solum fid.e et omnis et ita factis Iura/ menti Supra quibus Datum Roma hac die/ 27. Aprilis 1771./ Ita est Placensinus de Montibus Causarum/ Curia Camere Apostoli Not.o/... Fran.cus de Heio Causarum Curia/ Cam. Aplicus. Gnlis Auditor".

${ }^{14}$ En el recto del siguiente folio se puede leer: "Universis fidem facimus verboque veritatis resta/ mur supradictum D. Placensinum de Montibus/ esse Not.m pub.cum curis ac Tribunalis/ nostri, legalem collegialem, authenticum/ ac fide digna eiusque scripturis tam/ publicis quam privatis adhibiram semper/ fuisse ac de presenti quoque plenam indu/ biamque adhibiti fidem in iudicio, et/ extra. Datum Roma ex Magna Curia/ Innocenziana hac die 27: Aprilis 1771/ Ita est Clemens Iacobutius/ Caus. Cur. Cam. Aplic Not/ Ita est Ioes Alexander Paleanus/ Causanno Curia Camerae Aplic.e Not./ Ita est Leonardus Antonius Pace/ Causarum Curiae Camerae Apostolice Notarius".

15 FrAnÇOIS, 2011: 15.

16 Moroni Romano, 1856: 105-168.

17 Moroni Romano, 1856: 44. En la primera planta del palacio de Montecitorio se dispusieron las oficinas y las cancillerías de los tribunales civiles, en las plantas superiores el auditor general de la Reverenda Camera Apostólica, los tribunales de primera instancia, el tesorero general de la Reverenda Camera Apostólica y sus secretarías, así como el tribunal criminal del auditor. En la loggia central se celebraba, cada quince días, la extracción de los premios de la lotería lo que contribuía a aumentar la liquidez de la economía pontificia. La Curia Innocenziana siguió funcionando con regularidad hasta el año 1848, en que el auditor general de la Reverenda Cámara Apostólica dejó de tener su propio tribunal y abandonó la Curia Innocenziana como residencia.

18 Sant'Andrea delle Fratte, stati delle anime, 1751, Archivio Storico del Vicariato, Roma (ASV): "Strada nova ritorno./ del sig. Conte Antanori./ 2./ Sig. Piacentino Monti da Sonnino Not.o 50./ Lucia Puglese da Monticelli mog.e 38./ Pietro fig.o va sotto 11./ Anton Gius.e fig.o 3./ Vincenzo fig.o Marzo 50. 1./ Pietro figlio di Piacentino 11./ Bartolomea Vannone da Farnese zit.a s. 18./ Angela da S. Vite (...) 23".

19 Lodolini, 1956. El Auditor Camerae estaba inicialmente dividido en diez uffici aunque, en 1692 coincidiendo con el pontificado de Inocencio XII pasaron a ser cinco y, a lo largo de la primera mitad del siglo XIX, se redujeron gradualmente hasta convertirse primero en tres y, más tarde, en dos. Finalmente, desde el año 1856 hasta el 1871, quedó tan sólo el ufficio VI. Si bien es cierto que, de manera general, las tipologías documentales realizadas por los

Arch. esp. arte, LXXXVII, 346, ABRIL-JUNIO 2014, 109-118 ISSN: 0004-0428, eISSN: 1988-8511, doi: 10.3989/aearte.2014.08 
poseer un despacho por su cuenta en el que se ocupaban de otras comisiones ajenas al desarrollo a las necesidades de la Curia ${ }^{20}$.

\section{Peculiaridades e interrogantes sobre la presencia de Adán y Eraso en el Auditor Camerae}

En la parte inferior del recto del folio del documento escrito por Manuel Eraso y firmado también por Juan Adán, el notario Piacentino Monti escribe la frase "In Nomine Domini Amen/ Fidem facio per presentes Causarum Curia Camerae", que sirve para enlazar el contenido del recto con el del verso de ese folio. A lo largo del verso del mismo, el notario romano da fe de que la firma que aparece en el recto es, efectivamente, la de los dos artistas aragoneses y certifica que las personas que se encuentran en el lugar en que se está realizando la documentación que aquí nos ocupa son, sin lugar a dudas, Juan Adán y Manuel Eraso. En el recto del segundo folio, tres notarios del Auditor Camerae, Clemens Iacobutius del oficio $\mathrm{II}^{21}$, Leonardus Antonius Pace del oficio III $^{22}$ y Iohanes Alexander Paleanus del oficio VII ${ }^{23}$, acreditan, por su parte, que Monti es, a todos los efectos, un notario de la Curia Cameral.

El documento suscrito por Monti, lo que Adán y Eraso denominaron "las diligencias de libertad" practicadas en Roma, suscita algunas dudas y reflexiones. La primera de ellas es sobre la razón que impulsó a ambos a apelar a un notario del Auditor Camerae para prestar declaración y no se dirigieron a uno de los treinta notarios capitolinos, algo mucho más habitual entre los artistas españoles presentes en la ciudad eterna. Asimismo, a partir de esta cuestión, es posible plantear una pregunta ulterior: ¿cuál era el fundamento en que se apoyaba Monti para refrendar con tal fuerza la declaración de estos dos jóvenes?24

Estos interrogantes se podrían resolver suponiendo la presencia de un personaje relevante del mundo eclesiástico bajo cuya tutela, Eraso y Adán, y paralelamente Goya, podrían haber llegado a la Curia Innocenziana. Se podría incluso imaginar la intercesión de monseñor Azpuru (Zaragoza, 1713-Roma, 1772), embajador de España que, de manera evidente, había apoyado ya a Era$\mathrm{so}^{25}$ y a Adán ${ }^{26}$, sobre todo para que a ambos se les concediesen sendas pensiones de la Real Academia de Bellas Artes de San Fernando para permanecer en Roma.

notarios del Auditor Camerae no difieren en demasía de las que se hacían en otros notarios, hay que puntualizar que, en la documentación notarial del Auditor Camerae que ha llegado hasta nosotros, se constata la presencia, con cierta frecuencia, de inventarios de las familias nobles romanas y de muchos cardinales, así como de testamentos o quirógrafos.

${ }^{20}$ Lodolini, 1976: 237-332. Lodolini, 1992: 19-37. Además del Auditor Camerae, existía en Roma el colegio de los treinta notarios capitolinos, denominados de esta manera por tener su sede en el tribunal del Campidoglio en el palacio de los Conservadores. Éste fue constituido el 29 de diciembre de 1586 por voluntad de Sixto V (GrotTAMARE, 1521-Roma, 1590).

21 François, 2011: 11. Clemens Iacobutius estuvo activo entre los años 1747 y 1777.

22 FranÇOIS, 2011: 12. Activo en el Auditor Camerae entre 1767 y 1784.

${ }_{23}$ François, 2011: 16. Iohanes Alexander Paleanus estuvo vinculado al Auditor Camerae entre 1767 y 1773.

24 Entre la documentación conservada de Monti, no he conseguido encontrar otros documentos anteriores realizados por Eraso y Adán que avalen un conocimiento prolongado y certero de los dos jóvenes artistas aragoneses por parte del notario.

25 Junta ordinaria, 16 de febrero de 1766, Juntas particulares, ordinarias, generales y públicas, 1757-1769, Archivo de la Real Academia de Bellas Artes de San Fernando, Madrid (ARABSF), sig. 3/82.

${ }^{26}$ Junta ordinaria, 4 de octubre de 1767, Juntas particulares, ordinarias, generales y públicas, 1757-1769, ARABSF, sig. 3/82.

Arch. esp. arte, LXXXVII, 346, ABRIL-JUNIO 2014, 109-118

ISSN: 0004-0428, eISSN: 1988-8511, doi: 10.3989/aearte.2014.08 
Otro factor que también podría justificar la intercesión de Tomás de Azpuru y el acceso de Adán y Eraso a un ambiente restringido como el del Auditor Camera podría ser la prisa. Es probable que el viaje de Francisco de Goya, que había de llevar consigo el documento realizado ante Monti, se anticipase, es decir, que algún hecho inesperado obligase al pintor aragonés a salir de Roma. Podría ser el mismo imprevisto que impidió que esperase el veredicto del concurso de la Reale Accademia di Belle Arti de Parma al que había concurrido con la obra Aníbal que por primera vez miró Italia desde los Alpes (1771, Fundación Selgas-Fagalde, Cudillero) y a la que acompañaba una carta fechada el 20 de abril. Esto significa, además, que el documento firmado por Monti es el último del que tenemos conocimiento, hasta el momento, en que se refiere la permanencia de Goya en Italia.

Asimismo, el hecho de que Goya pidiese a sus amigos que firmasen un documento notarial que corroborase que, durante su soggiorno romano, no había contraído matrimonio, podría dar lugar a la sospecha de que el joven pensaba casarse en una fecha no muy lejana a abril de 1771, aunque el enlace se celebrase en 1773. Quizá, las comisiones artísticas encargadas a Francisco de Goya entre los años 1771 y 1772, es decir, los frescos del Coreto de Nuestra Señora del Pilar y las pinturas murales de la cartuja de Aula Dei, representasen una extraordinaria oportunidad para el despegue de su carrera, lo suficiente como para retrasar su boda.

Esta reflexión nos lleva a imaginar, de nuevo, que la prisa hubiese sido lo que propició que el documento firmado ante Monti se confeccionase de una manera sencilla pero eficaz, como un atestado que no olvida los datos esenciales, pero que no se detiene demasiado en las vicisitudes de la estancia de Goya en Italia o en los vínculos que le unen a los dos testigos. Incluso, se podría creer que, cuando se llevó a cabo el documento ante el notario, Goya no tuviese una demora fija en Roma o que el viaje de regreso a España fuese realmente inminente.

Otro aspecto insólito del documento realizado por Monti es que su testimonio queda refrendado, a su vez, por otros tres notarios, como si alguien pudiese tener alguna duda de la veracidad de la identidad del notario que firma el documento de Adán y Eraso. Estos tres notarios, con su palabra, dan fe de que, efectivamente, Monti es quien dice ser y desarrolla su actividad en el Tribunal del Auditor Camerae. Si bien es cierto que se trata de un documento que habrá de ser útil en territorio español, en donde el notario que lo suscribe probablemente no era conocido para quien lo habría de emplear, resulta cuanto menos excesivo el celo con que se garantiza la potestad de Monti en calidad de notario.

Esta última peculiaridad del documento suscita, a su vez, otras dos sospechas: ¿es posible que algún aspecto de la conducta de Goya durante su estancia en Roma pudiese hacer dudar, en algún momento, de la veracidad de la documentación que alegaba o de sus testimonios? O por el contrario, ¿un documento elaborado con tanto esmero, en el que se cuidan todo tipo de detalles, se podría deber a un trato de favor, o al hecho de que Goya tuviese relevantes contactos en la ciudad eterna?

\section{La relación de Goya con Juan Adán y con Manuel Eraso y su incidencia en el desarrollo de su estancia en Roma}

La insistencia con que Adán y Eraso afirman haber conocido a Goya durante su permanencia en la ciudad eterna y la carencia, hasta el momento, de un documento que precise en qué lugar de Roma pudo vivir el joven pintor, son factores que invitan a intentar describir, con mayor claridad de lo que se ha hecho hasta el momento, el que podría haber sido el ambiente frecuentado por el pintor aragonés a partir de la relación con sus dos paisanos.

En el intervalo de tiempo en que Goya estuvo en Italia, es decir, entre 1769 y 1771, Juan Adán y Manuel Eraso se alojaron en la parroquia de Sant'Andrea delle Fratte, verdadero centro 
neurálgico de Roma. Juan Adán se estableció en $1770^{27}$ en la casa de un pastelero al que alquilaba una habitación, mientras que en $1771^{28}$ lo hizo en esta misma calle, aunque en compañía de Gabriel Durán (Vich, 1747ca-Roma, 1806), al que algunos años después le habría de unir una relación de parentesco, puesto que ambos se casaron con dos hijas de Filippo della Valle (Florencia, 1698-Roma, 1768), Adán con Violante ${ }^{29}$ y Durán con Petronilla ${ }^{30}$.

En el caso de Manuel Eraso, su vida en aquellos años se desarrolló en el palacio de España ${ }^{31}$, en donde también habitaba un personaje aragonés llamado Francisco Claveras que había estado anteriormente en la iglesia-hospital de Santa Maria di Monserrato degli Spagnoli ${ }^{32}$ y que podría haber motivado o facilitado el traslado de Eraso al palacio de España. Es posible que Eraso, como podrían haber hecho también en algún caso Goya y Adán, recurriese, en momentos de especial carestía, a la iglesia-hospital de Santa Maria di Monserrato degli Spagnoli, vinculada a la corona aragonesa, en donde podría haber residido durante un periodo y, posteriormente, gracias a la intercesión de Claveras y de Azpuru, se habría mudado al palacio sito en la plaza de España ${ }^{33}$.

La relación de Eraso con Azpuru está clara, tal y como hemos comentado anteriormente, y queda subrayada por el hecho de que Manuel Eraso abandonó el palacio de España precisamente tras la Pascua del año 1772, en que falleció Tomás Azpuru ${ }^{34}$. Por lo que respecta a los vínculos que unen a Francisco Claveras con Azpuru, resulta particularmente indicativo que el clérigo

27 Sant'Andrea delle Fratte, stati delle anime, 1770, ASV: "=Strada Fratina/=1=/ =Del Marchese Consalvi=/ Giuseppe Moretti Pasticciere 43 / Anna Muceri Moglie 35 / =Piggionante= / Giovanni Adami scultore 24".

${ }_{28}$ Sant'Andrea delle Fratte, stati delle anime, 1771, ASV: “2. / d: gabriele durán spagnolo 30/ d: giovanni Adami $24 "$.

29 Santa Susanna, Liber matrimoniorum, 3, 1774-1810. ASV: Juan Adán y Violante della Valle se casaron el 20 de marzo de 1776 y realizaron las interrogationes, que no se conservan, en el notario capitolino Michelangelus Clementi.

30 Santa Susanna, Licenze matrimoniali, 4, 1761-1790. ASV: La licencia del matrimonio de Gabriel Durán y Petronilla della Valle fue firmada el 12 de octubre de 1776 por el párroco de Santa Susanna, Paolo Altobelli, estando ambos adscritos en ese momento a dicha parroquia, según se indica en el documento. Santa Susanna, Liber matrimoniorum, 3, 1774-1810, ASV. El enlace tuvo lugar en la parroquia de Santa Susanna el 18 de noviembre de 1776, para lo que los contrayentes aportaron unas interrogationes realizadas en la notaría de Michelangelus Clementi.

31 Sant'Andrea delle Fratte, stati delle anime, 1770, ASV: "=Palazzo Reggio= / (...) D. Emanuelle Eraso Pittore 28". Sant'Andrea delle Fratte, stati delle anime, 1771, ASV: "Palazzo Reggio di Spagna / (...) D. Emmanuele Eraso Pittore 29". Sant'Andrea delle Fratte, stati delle anime, 1772, ASV: "Palazzo Regio di Spagna/ (...) d. emmanuele eraso, pittore 30".

32 Santa Caterina della Rota, stati delle anime, 1768, ASV: "Casa ed Ospedale di Monserrato/ Rev. Sig. D. Michele Gascon di Aragona Rettore 49 / Rev. Sig. D. Giuseppe Carbó da Valenza Magiorduomo 52 / Rev. Sig. D. Giovanni Dominech da Majorica 45 / Rev. Sig. D. Giovanni Miglia da Ager Sagrestano 57 / Rev. Sig. D. Giuseppe Porta Catalano 58/ Rev. Sig. D. Agostino Bux di Aragona 60 /Rev. Sig. D. Michele Pujale Romo. 32 / Rev. Sig. D. Felice Grua Catalano 50 / Chierici. Sig. D. Tomaso Lopis da Valenza 59 / Sig. D. Ignacio Peyra Catalano 42 / Sig. D. Vincenzo Gafió da Valenza 60 / Sig. D. Francesco Claveras d'Aragona 35 / Sig. D. Francesco Ravegli da Catalogna 55 / Sig. Paolo Oliber da Majorica Cuoco". El libro de stati delle anime de Santa Caterina della Rota de 1769 no se conserva. Santa Caterina della Rota, stati delle anime, 1770, ASV: "Casa ed Ospedale di Monserrato. / Riv. Sig. D. Michele Gascon di Aragona Rettore 51 / Riv. Sig. D. Giuseppe Carbó da Valenza Maggiorduomo 54 / Riv. Sig. D. Miglia da Ager Sagristano 59 / Riv. Sig. D. Giuseppe Porta Catalano 60 / Riv. Sig. D. Felice Brua Catalano 52 / Riv. Sig. D. Giacomo Vincenzo da Majorica 51 / Riv. Sig. D. Giacchino Valles d'Aragona 60 / Riv. Sig. D. Michele Puiale Romo. Mro di Cerimonie 34 / Chierici Sig. D. Tomaso Lopis da Valenza 61/ Sig. D. Ignazio Peyra Catalano 44 / Sig. D. Vincenzo Gafió da Valenza 62 / Sig. D. Fran. co Claveras d’Aragona 37 / Sig. D. Francesco Ravegli da Catalogna 57 / Sig. Francesco Vincenti da Valenza Cuoco 35".

33 Sant'Andrea delle Fratte, stati delle anime, 1771, ASV: "Palazzo Reggio di Spagna / Ilmo Monsignore d Tomasso Aspurru / d. Patrizio secretario / d. Salvatore Rodriguez / d. Giuseppe Garzes 52 / Sign.a Angela Conop. Moglie 42 / Giuseppe figlio 7 / Emmanuele figlio 5 / Barbara figlia 2 / Apollonia Guerini, zitella serva 30 / Cristofaro servo 37 / d. Francesco Claveras 38 / d. Emmanuele Mendizabba 47 / Signora Casavina faccensi moglie 39”.

34 Sant'Andrea delle Fratte, morti, 1740-1810, ASV. Tomás Azpuru falleció el 7 de julio de 1772 a la edad de 59 años. Su cadáver fue velado y enterrado en la iglesia de Santa Maria ai Monti.

Arch. esp. arte, LXXXVII, 346, ABRIL-JUNIO 2014, 109-118

ISSN: 0004-0428, eISSN: 1988-8511, doi: 10.3989/aearte.2014.08 
se fuese de Italia desde Nápoles en febrero de ese mismo año, es decir, en una fecha no muy distante a la muerte del embajador tras una enfermedad ${ }^{35}$.

Aunque Goya no hubiese vivido en la parroquia de Sant'Andrea delle Fratte, sus dos amigos Eraso y Adán sí lo hicieron y por ello, pudo frecuentar este espacio y, con bastante probabilidad, debió estar familiarizado con el entramado de amistades tejido por aquellos durante sus años romanos. Por esta razón, cabe recordar que, en la parroquia de Sant'Andrea delle Fratte, se encontraban en 1770 algunos amigos de Eraso y Adán; uno de ellos es Angelo Antonio Brizzolari ${ }^{36}$ (Carrara, 1754-Roma, 1772) ${ }^{37}$ que había vivido con Adán en 1768 y $1769^{38}$ en la parroquia de Santa Susanna ${ }^{39}$ y que había compartido con él la experiencia de ser premiado en los concursos de la Scuola del Nudo.

En marzo de 1767, Adán ganó el primer premio de escultura, mientras que el segundo fue para Brizzolari ${ }^{40}$; en marzo de 1768, bajo la dirección de Francisco Preciado de la Vega (Sevilla, 1712-Roma, 1789), el turiasonense recibió el segundo premio de la primera clase de la Scuola del Nudo, mientras que Brizzolari se alzó con el segundo galardón de escultura ${ }^{41}$. En marzo de 1769, de nuevo bajo la dirección de Preciado, Brizzolari obtuvo el segundo premio de escultura, en septiembre de ese mismo año Adán resultó galardonado con el primer premio de escultura ${ }^{42}$; en marzo de 1770, Brizzolari recibió el segundo premio de escultura ${ }^{43}$. No debiéramos excluir que el escultor de Carrara, en virtud a su estancia en la parroquia de Santa Susanna, en la que vivía de forma estable el tutor de los pensionados españoles Preciado de la Vega, se pudiese haber formado bajo la tutela del sevillano beneficiándose de la posición que aquel tenía en el ambiente académico romano.

Otro de los galardonados en la Scuola del Nudo fue Francesco Antonio Franzoni (Carrara, 1734-Roma, 1818 $)^{44}$, que estaba establecido en la misma casa de Brizzolari en 1770, en la parroquia de Sant'Andrea delle Fratte, muy cerca del domicilio de Juan Adán y del palacio de España en que vivía Eraso. Es probable que Franzoni, un escultor joven pero que empezaba ya a finales

35 Segreteria di stato di casa reale, 1259, fol. 19v, passaporti, Archivio Storico di Napoli: "13 de febrero de 1772, D.n Francesco Claveras spagnolo con bigl.o del Min.ro di Spagna”.

36 Sant'Andrea delle Fratte, stati delle anime, 1770, ASV: "=Del Vicolo della Purificazione/ =de Bennani di Firen$\mathrm{ze}=/=1=/$ Angelo Antonio Brizolai scultore 25/ Pietro Fratello 22/=2= Fran.co Antonio Franzioni Scultore 35/ Giuseppe fratello 21 ".

37 Sant'Andrea delle Fratte, Morti, 1758-1813, ASV: El fallecimiento de Angelo Antonio Brizzolari tuvo lugar el 13 de junio de 1772, cuando tenía tan sólo 28 años de edad.

38 Santa Susanna, stati delle anime, 1768, ASV: "piazza Barbarini/ 325 casa al 3/S. Alesandro Cruze 25/S. Gio. Adamo 22/S. Angelo Baizigo can 25". Santa Susanna, stati delle anime, 1769, ASV: "piazza Barberini/328 casa al 3/ Gio Adamo 23/Angelo Ant. ${ }^{\circ}$ Brizzo 25".

39 De la Cruz Alcañiz, García Sánchez, 2009: 69-92. De la Cruz Alcañiz, García Sánchez, 2010: pp. 665-670.

40 Nome e cognome di tutti i premiati alla Scuola del Nudo dall'Anno 1754 al 1848; coll'indicazione del professore direttore della scuola. Sigue un elenco alfabetico degli alumni che hanno frequentato la Scuola in un'anno, Archivio dell'Accademia di San Luca, Roma (AASL), A33b1, fol. 14r.

${ }^{41}$ Nome e cognome..., AASL, 33b1, fol. 15r.

42 Nome e cognome..., AASL, 33b1, fols. 16r-16v.

43 Nome e cognome..., AASL, 33b1, fols. 16v-17r.

44 Dizionario biografico degli italiani, 1998: 283-287. Francesco Antonio Franzoni nació en Carrara en 1734 y murió en Roma en 1818. En un primer momento se formó en la ciudad toscana, hasta que, en 1758, es registrado por primera vez en Roma viviendo en una locanda con Michele Baratti, en la via della Purificazione. A finales de los años sesenta, junto a Guillaume-Antoine Grandjacquet (Reugney, 1731-Roma, 1801) y Lorenzo Cardelli restauró la colección de antigüedades de Giovanni Battista Piranesi y, entre los años 1769 y 1774, recibió diversos pagos por su intervención en la iglesia romana de Santa Caterina dei Senesi. Posteriormente, los conocimientos adquiridos en la Sala de los animales del Museo Pio-Clementino gracias a la restauración de un significativo número de sus esculturas le permitieron cosechar una experiencia que se concretó en la colaboración, años más tarde, con Vicenzo Pacetti (Castel Bolognese, 1746-Roma, 1820), quien también dedicó buena parte de su trayectoria artística a la restauración de piezas clásicas.

Arch. esp. arte, LXXXVII, 346, ABRIL-JUNIO 2014, 109-118 ISSN: 0004-0428, eISSN: 1988-8511, doi: 10.3989/aearte.2014.08 
de los años sesenta afirmarse en el panorama artístico romano -de hecho, por aquel entonces se ocupaba de la restauración de la colección de piezas clásicas de Giovanni Battista Piranesi (Mogliano Veneto, Treviso, 1720-Roma, 1778)- formase parte, aunque fuese ocasionalmente, del grupo de amistades de los tres artistas aragoneses.

Naturalmente, Goya debió conocer bien el círculo de personalidades y artistas españoles que vivía en la ciudad eterna entre 1769 y 1771, empezando por Francisco Preciado de la Vega ${ }^{45}$ y por Tomás Azpuru, sobre cuyo apoyo a los aragoneses no es necesario insistir. Asimismo, pudo frecuentar con asiduidad a Alejandro de la Cruz (Salamanca, 1738-Madrid, 1811) ${ }^{46}$, durante algún tiempo establecido en la parroquia de Santa Susanna ${ }^{47}$, y que, como Adán y Eraso, disfrutaba de una pensión extraordinaria obtenida gracias al apoyo de Anton Raphael Mengs (Bohemia, 1728Roma, 1779 $)^{48}$.

Además, es bastante probable que el de Fuendetodos entrase en contacto con Domingo Álvarez Enciso (Mansilla de la Sierra, Burgos, 1737-Jerez de la Frontera, Cádiz, 1800) ${ }^{49}$ quien nunca debió romper sus vínculos con la comunidad artística española presente en la ciudad eterna. Enciso regresó a España en 1766 cuando concluyó su pensión, aunque se registra nuevamente en la ciudad eterna en 1767, en donde contrajo matrimonio en la iglesia de Sant'Eustachio con la romana Angela Morosini ${ }^{50}$.

\section{BIBLIOGRAFÍA}

Ansón Navarro, Arturo, Goya y Aragón. Familia, amistades y encargos artísticos, col. Mariano de Pano y Ruata, n. ${ }^{\circ}$ 10, Zaragoza, Caja de Ahorros de la Inmaculada de Aragón, 1995.

Ansón Navarro, Arturo, "La formación artística de Goya y la etapa zaragozana, 1759-1774", en Luna, Juan José (comisario), Goya. 250 aniversario, cat. exp., Madrid, Museo del Prado, 1996, pp. 57-63.

Azcue Brea, Leticia, La escultura en la Real Academia de Bellas Artes de San Fernando. Catálogo y estudio, Madrid, Real Academia de Bellas Artes de San Fernando, 1994.

Banda y Vargas, Antonio de la, De la Ilustración a nuestros días. Historia del Arte en Andalucía, vol. 8, Sevilla, Ediciones Gever, 1991.

Calvo Carretero, Rebeca, El escultor Juan Adán y su entorno familiar, en Goya y su contexto, Actas del Seminario Internacional, Zaragoza, Institución Fernando el Católico, 2013, pp. 411-428.

Contento Márquez, Rafael, "Formación del buen gusto", en Formación del buen gusto. Dibujos de pensionados en Roma (1752-1786), cat. exp., Madrid, Facultad de Bellas Artes, 1995, pp. 31-34.

De la Cruz Alcañiz, Cándido, "Entre la fortuna y el olvido. La actividad pictórica del pensionado romano Domingo Álvarez (1739-1800)", Atrio, 13 y 14, 2007/2008, pp. 53-70.

45 Si se compara la letra de la carta que Goya envió a la Reale Accademia di Belle Arti de Parma para acompañar el cuadro con el que optaba al premio con la documentación escrita por Preciado de la Vega en las Congregazioni académicas de aquellos años en que fue secretario de la Accademia di San Luca, caben pocas dudas de que la primera fue escrita por el proprio Preciado. La brevedad de la estancia que Goya pasó en Italia, hace que sea lógico imaginar que el italiano del pintor aragonés fuese muy limitado, insuficiente para escribir una carta correcta como la que acompañó al cuadro que presentó al concurso de la ciudad emiliana.

46 Contento, 1995: 31-34. De la Cruz Alcañiz, García SÁnchez, 2008: 107-120. Sureda, 2008: I 257. Urrea, 2006: 198-199.

47 Santa Susanna, stati delle anime, 1768-1771, ASV.

48 Solicitud de académico de mérito, 1 de mayo de 1768, ARABSF, leg. 5-174-1.

49 Banda y Vargas, 2008: 63. De la Cruz Alcañiz, 2007/2008: 53-70. Pérez Sánchez, 1986: 448. Sureda, 2008: I 249-250. URREA, 2006: 182, 222-224.

50 Sant'Eustachio, Matrimoni, 1665-1784, ASV, fol. 309v. En 1782, Domingo Álvarez seguía viviendo en Roma en la strada Paolina verso la piazza di Spagna, en la parroquia de San Lorenzo in Lucina en compañía de su esposa y de su hijo Camillo de 15 años (San Lorenzo in Lucina, stati delle anime, 1782, ASV).

Arch. esp. arte, LXXXVII, 346, ABRIL-JUNIO 2014, 109-118

ISSN: 0004-0428, eISSN: 1988-8511, doi: 10.3989/aearte.2014.08 
De la Cruz Alcañiz, Cándido, García Sánchez, Jorge, “Alejandro de la Cruz. Un discípulo de Mengs en Roma", Goya, 323, 2008, pp. 107-120

De la Cruz Alcañiz, Cándido, García Sánchez, Jorge, "L’Accademia romana di Francisco Preciado de la Vega in piazza Barberini e gli artisti spagnoli del Settecento", Bollettino d'arte, 7, 94, 2009, pp. 91-102.

De la Cruz Alcañiz, Cándido, García Sánchez, Jorge, "Piazza Barberini: a Spanish Artists' District in Eighteenth-Century Rome”, Burlington Magazine, 152, 2010, 1291, pp. 665-670.

Dizionario biografico degli italiani, 50, Catanzaro, Arti grafiche Abramo, 1998.

François, Achille, Repertorio dei Notari Romani dal 1348 al 1927, edición de Romina de Vizio, Roma, Fondazione Marco Besso, 2011.

Gallego, Raquel, "Francisco de Goya: vivir en Roma”, en Sureda, Joan (comisario), Goya e Italia, cat. exp., Zaragoza, Museo de Zaragoza, Madrid, Turner, 2008, pp. 45-59.

Gallego, Raquel, "Metodología para el estudio del panneggio en el Cuaderno italiano: fundamentos teóricos, espacios académicos y su puesta en práctica en el taccuino goyesco", en Cartografias visuales y arquitectónicas de la modernidad, Barcelona, Publicacions i Edicions de la Universitat de Barcelona, 2011, pp. 47-60.

Ibáñez Pérez, Alberto C., Historia de la Academia de Dibujo de Burgos, Burgos, Publicaciones de la Excma. Diputación de Burgos, 1982.

Lodolini, Armando, Archivio di Stato di Roma, Inventario dell'archivio dello Stato Pontificio, Roma, Associazione Bancaria Italiana, 1956.

Lodolini, Elio, "Formazione dell'Archivio di Stato di Roma”, Archivio de la Società di Storia Patria, XCIX, 1976, pp. 237-332.

Lodolini, Elio, Gli Istituti archivistici romani en L'Archivio di Stato di Roma, Roma, Nardini editore, 1992.

López Ortega, Jesús, "El expediente matrimonial de Francisco de Goya", Boletín del Museo del Prado, 44, 2008, pp. 62-68.

Mena Marqués, Manuela B., Urrea, Jesús, El Cuaderno italiano, 1770-1786: los orígenes del arte de Goya, Madrid, Museo del Prado, 1994.

Mena Marqués, Manuela B., Goya: el viaje interior en Borrás Gualis, Gonzalo M.: El arte en el siglo de las luces, Madrid, Galaxia Gutenberg, 2010, pp. 337-376.

Moroni Romano, Gaetano, Dizionario di erudizione storico-eclesiastica, vol. LXXX, Venecia, Tipografia Emiliana, 1856.

Navarrete Prieto, Benito, "Francisco de Goya en San Nicolás de los Loreneses en Roma, 1770", Archivo Español de Arte, 75, 2002, pp. 293-296.

Ona González, José Luis, Goya y su familia en Zaragoza. Nuevas noticias biográficas, Zaragoza, Institución Fernando el Católico, 1997.

Pardo Canalis, Enrique, Escultores del siglo XIX, Madrid, Instituto Diego Velázquez de Arte, 1951.

Pardo Canalis, Enrique, "Las estatuas de San Pedro Arbués y Santa Isabel, por Juan Adán”, Goya, 70, 1965/66, p. 197.

Pardo Canalis, Enrique, “La Venus de Juan Adán”, Goya, 102, 1971, pp. 435-436.

Pérez Sánchez, Alfonso, Historia del dibujo en España. De la Edad Media a Goya, Madrid, Cátedra, 1986.

Reuter, Anna, "Francisco de Goya en el extranjero: el viaje a Italia y el exilio en Francia", en Cabañas Bravo, Miguel, El arte español fuera de España, XI jornadas de arte, 2002, Madrid, Consejo Superior de Investigaciones Científicas, 2003, pp. 37-48.

Sureda, Joan (comisario), Goya e Italia, cat. exp., 2 vols., Madrid, Turner, 2008.

Tosini Pizzetti, Simona (comisaria), Goya e la tradizione italiana, cat. exp., Cinisello Balsamo, Silvana Editoriale, 2006.

Urrea, Jesús, “Goya en Italia: a propósito del Aníbal”, Boletín del Museo del Prado, 14, 1993, 32, pp. 59-66.

Urrea, Jesús, Relaciones artísticas hispano-romanas en el siglo XVIII, Madrid, Fundación de Apoyo a la Historia del Arte Hispánico, 2006.

Vaquero Peláez, Dimas, "El escultor Juan Adán, un turiasonense en el olvido”, Turiaso, vol. 10, 2, 1992, pp. 547-562.

Fecha de recepción: 29-X-2012

Fecha de aceptación: 06-V-2013

Arch. esp. arte, LXXXVII, 346, ABRIL-JUNIO 2014, 109-118 ISSN: 0004-0428, eISSN: 1988-8511, doi: 10.3989/aearte.2014.08 